\title{
Raman scattering by coupled plasmon-LO phonons in InN nanocolumns
}

\author{
S. Lazić , E. Gallardo , J.M. Calleja , F. Agulló-Rueda , J. Grandal , M.A. Sánchez-García , \\ and E. Calleja
}

Dept. Física de Materiales, Universidad Autónoma de Madrid, 28049 Madrid, Spain

Materials Science Institute of Madrid, CSIC, 288049 Madrid, Spain

ISOM and Departamento de Ingeniería Electrónica, ETSIT, Universidad Politécnica de Madrid, 28040 Madrid, Spain

\begin{abstract}
Raman measurements on high quality, relaxed InN nanocolumns grown on $\mathrm{Si}(001)$ and $\mathrm{Si}(111)$ substrates by plasmaassisted molecular beam epitaxy are reported. A coupled LO phonon-plasmon mode around $430 \mathrm{~cm}^{1}$, together with the uncoupled LO phonon appears in the nanocolumnar samples. The coupled mode is attributed to spontaneous accumulation of electrons at the lateral surfaces of the nanocolumns, while the uncoupled phonon originates from their inner part. Infra-
\end{abstract}

1 Introduction The small band gap and effective mass of $\mathrm{InN}$ as compared to other group III-nitrides, will potentially enlarge the range of applications of nitrides to high speed device applications working in the visible and infrared spectral regions. The main obstacles to progress in this direction are the growth difficulties of InN-based structures mainly due to the $\mathrm{InN}$ low dissociation temperature, and the poorer knowledge of InN fundamental parameters. Besides, InN layers systematically show high electron concentrations, whose origin (unintentional doping during growth, interface effects or surface accumulation) is still under discussion. Free electron oscillations couple to the longitudinal optical (LO) phonons producing mixed phonon-plasmon excitations $\left(\mathrm{L}_{+}, \mathrm{L}\right.$.) Coupled modes in InN films have been studied by Raman scattering infrared (IR) reflectance and IR ellipsometry and Raman scattering measurements in InN nanocolumns (NC) have also been reported [9]. Several authors attribute the mode observed at the $A_{1}(\mathrm{LO})$ frequency to a $\mathrm{L}$. mode shifted to high energies by wavevector non-conserving scattering processes This explanation requires large scattering wavevectors, which imply significant crystalline red retlectance measurements confirm the presence of electrons in the nanocolumns. The electron density in the accumulation layer depends on the growth temperature and is sensitive to exposure of $\mathrm{HCl}$. Our results indicate that accumulation of intrinsic electrons occurs not only at the polar surfaces of InN layers, but also on non-polar lateral surfaces of $\mathrm{InN}$ nanocolumns. Its origin is attributed to an In-rich surface reconstruction of the nanocolumns sidewalls.

disorder. Recent work

indicate the presence of an intrinsic electron accumulation layer on the polar surfaces of InN films, due to the low energy of the conduction band minimum of InN and the pinning of the Fermi level at ionized surface donor states.

In this paper we present Raman and IR reflectance measurements on both compact and $\mathrm{NC}$ InN high quality samples. The compact samples display the allowed $\mathrm{E}_{2}$ and $\mathrm{A}_{1}$ (LO) modes in backscattering from the z-plane. However, the NC samples show the LO mode at the $\mathrm{E}_{1}$ frequency, together with an L. coupled mode in the 425-450 $\mathrm{cm}^{-1}$ range. The corresponding electron concentrations vary from 2.5 to $6.0 \times 10^{18} \mathrm{~cm}^{-3}$ depending on the growth temperature and can be altered by surface chemical treatments. Our results indicate that electron concentration occurs also at the non-polar NC sidewalls, giving rise to coupled modes, while the uncoupled LO phonon originates from the regions inside the $\mathrm{NC}$. The origin of the surface electron accumulation layer is attributed to a temperaturedependent In-rich surface reconstruction on the NC sidewalls. 
2 Experimental $\mathrm{InN}$ films were grown on $\mathrm{Si}(111)$ and $\mathrm{Si}(001)$ substrates by plasma-assisted molecular beam epitaxy (PAMBE). A $50 \mathrm{~nm}$ thick AlN buffer layer was used to optimize growth conditions. The III/V ratio was varied from $\mathrm{N}$-rich conditions (NC samples) to quasistoichiometry (compact samples). The growth temperature was varied between 450 and $480{ }^{\circ} \mathrm{C}$. The nanocolumns range from 80 and $150 \mathrm{~nm}$ in diameter depending on growth conditions. Scanning electron microscopy (SEM) and high resolution transmission electron microscopy (HRTEM) reveal the practical absence of defects and strain in the NC samples Raman scattering and IR reflectance measurements were carried out at room temperature, both in macro- and micro-configurations, using Argon and He-Ne laser for excitation and a charge coupled device detector. The spectra were taken in nominal backscattering configurations, both along the growth direction (c-axis): $z(x,-) \overline{\mathrm{Z}}$, and perpendicular to it: $x(y,-) \overline{\mathrm{x}}$ at the lateral sample surface (see left inset in Fig. 1).

3 Results and discusion Raman spectra taken in $z(x,-) \bar{z}$ scattering configuration are shown in Fig. 1 for a compact layer (a), and two $\mathrm{NC}$ samples grown at $460{ }^{\circ} \mathrm{C}$ (b) and $475^{\circ} \mathrm{C}$ (c) on $\mathrm{Si}(111)$. Spectra (a), (b) and (c) correspond to $632.8 \mathrm{~nm}$ excitation, while spectrum (d) is the same as (c) but with $514 \mathrm{~nm}$ excitation.

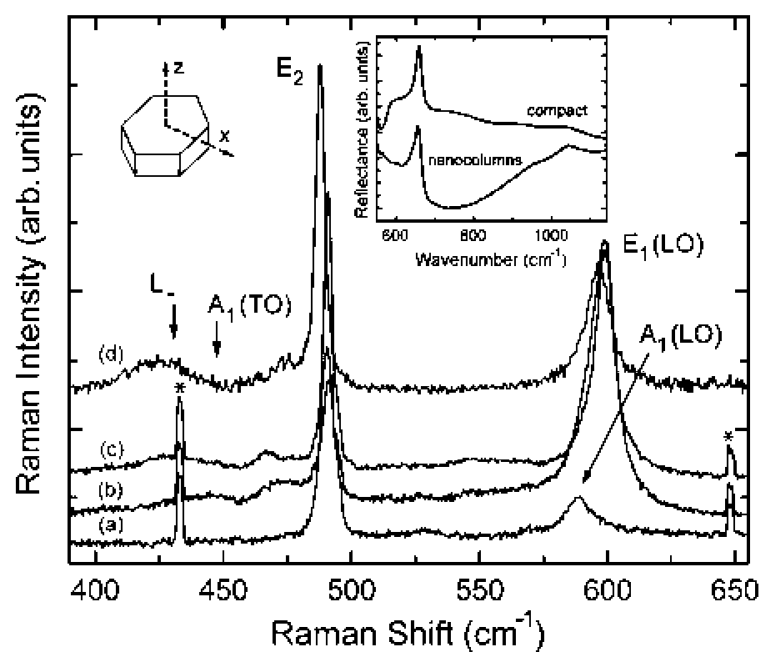

Figure 1 Macro-Raman spectra of several samples. The left inset shows the scattering directions relative to the crystal structure. The right inset displays the infrared reflectance spectra of the compact layer and the NC sample grown at $475^{\circ} \mathrm{C}$. Ne plasma lines are marked with asterisks.

The compact sample shows the allowed $\mathrm{E}_{2}$ and $\mathrm{A}_{1}(\mathrm{LO})$ modes. The small width $\left(4.0 \mathrm{~cm}^{-1}\right)$ of the non-polar $\mathrm{E}_{2}$ mode indicates the high crystalline quality observed by HRTEM [14]. The NC samples show also a narrow $E_{2}$ peak at the same frequency. However, the LO mode appears at the $\mathrm{E}_{1}(\mathrm{LO})$ frequency $\left(597 \mathrm{~cm}^{-1}\right)$, which is forbidden in this scattering geometry. This apparent failure of selection rules is attributed to the light scattering by the uneven height distribution of the $\mathrm{NC}$ and to light refraction at the NC sidewalls, resulting in the photons and LO phonons propagating nearly perpendicular to the c-axis. This is confirmed by micro-Raman measurements in nominal $x(y,-) \overline{\mathrm{x}}$ scattering configuration from the lateral surface of the samples excited at $632.8 \mathrm{~nm}$ (Fig. 2). In addition to the strong peak at $521 \mathrm{~cm}^{-1}$ of the Si substrate one observes the $\mathrm{E}_{1}(\mathrm{LO})$ phonon in the two samples. This shows that the light propagates essentially perpendicular to the NC sidewalls, irrespective of the nominal scattering geometry. The TO modes are observed in the low frequency region of Fig. 2 in both samples.

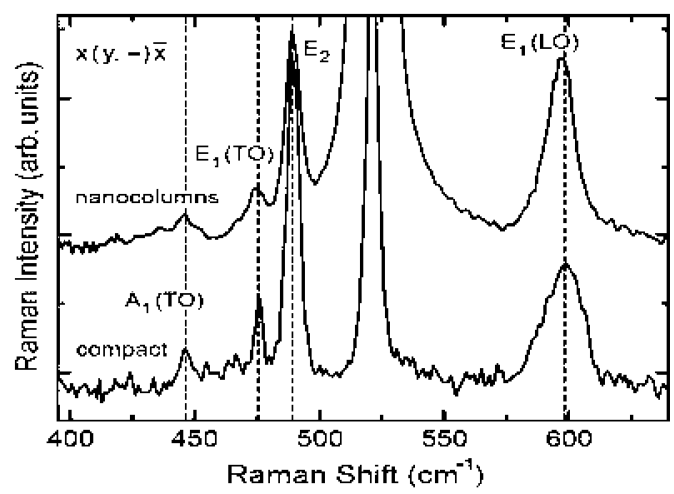

Figure 2 Micro-Raman spectra of samples (a) and (c) of Fig. 1 in $x(y,-) \overline{\mathrm{x}}$ scattering configuration.

The lateral surfaces of the $\mathrm{NC}$ being the main source of light scattering, we attribute the broad peak observed in the $425-450 \mathrm{~cm}^{-1}$ range in NC samples (Fig. 1) to the lowenergy branch L_ of the coupled LO phonon-plasmon mode arising from an electron accumulation layer at the lateral surfaces of the NC. The L mode is not observed in the compact layers, probably due to their comparatively low specific surface. The uncoupled $\mathrm{E}_{1}(\mathrm{LO})$ phonon arises from the inner part of the $\mathrm{NC}$. where the electron density is expected to be much smaller The intensity ratio of the $L_{\text {- }}$ to the $E_{1}(L O)$ peaks versus excitation wavelength supports this assignment. The ratio decreases by a factor of 4 from $514 \mathrm{~nm}$ to $632.8 \mathrm{~nm}$ excitation (Fig. 1(c) and (d)), corresponding to the smaller penetration depth of the 514 $\mathrm{nm}$ light. This difference in penetration depth could explain the small changes observed in the phonon frequencies. The presence of free electrons in the NC is confirmed by the infrared reflectance measurements shown in the right inset of Fig.1, where a minimum in the $700-800 \mathrm{~cm}^{-1}$ region is observed for the NC sample. The electron concentration corresponding to the observed $\mathrm{L}_{\text {- values can }}$ be estimated from the simplest model for the dielectric function Assuming an effective mass $\mathrm{m}^{*}=0.05 \mathrm{~m}_{0}$ the values of $\mathrm{n}$ for all samples studied are presented in Fig. 3 as a function of the growth temperature. 


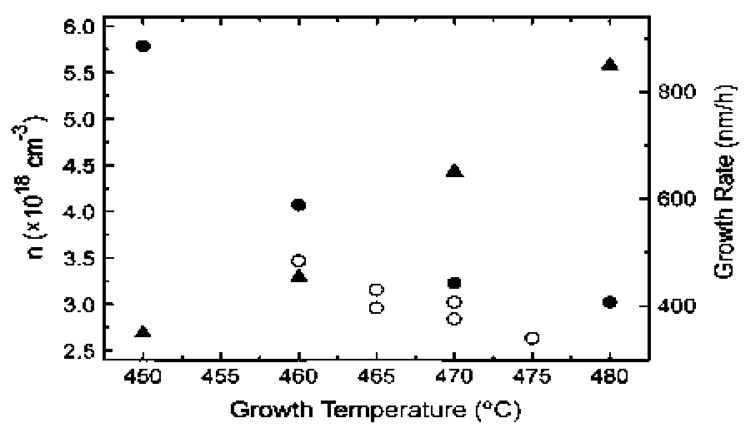

Figure 3 Electron concentration (circles) and growth rate (triangles) versus growth temperature for NC samples grown on $\mathrm{Si}(111)$ (open circles) and $\mathrm{Si}(001)$ (full circles) substrates.

The electron concentration decreases while the growth rate increases for increasing growth temperature. The higher growth rate observed at higher growth temperature is due to the faster In diffusion along the NC sidewalls. This implies a smaller number of In atoms present in the $\mathrm{NC}$ sidewalls as their speed increases at high temperatures. The mobile In atoms are frozen upon sample cooling at the end of the growth process. They are incorporated into the $\mathrm{NC}$ to form an In-rich surface layer responsible for the electron accumulation This accumulation should be proportional to the excess In atoms and consequently, it should be higher for lower growth temperatures, as observed.

The accumulation layer is also sensitive to the chemical environment of the NC. Fig.4 presents the temporal evolution of the electron concentration of a $\mathrm{NC}$ sample (measured again by the L- frequency) after dipping it in $\mathrm{HCl}$ for 1 minute and letting it dry in air. The initial density is $2.78 \times 10^{18} \mathrm{~cm}^{-3}$. The observed trend indicates a slight increase of the electron concentration followed by a slow recovery to close to the initial values after approximately 10 days in air. Although the chemical processes responsible for this behaviour are not clear yet, they could involve a transient change of the ionization state of surface donors, as in polar surfaces

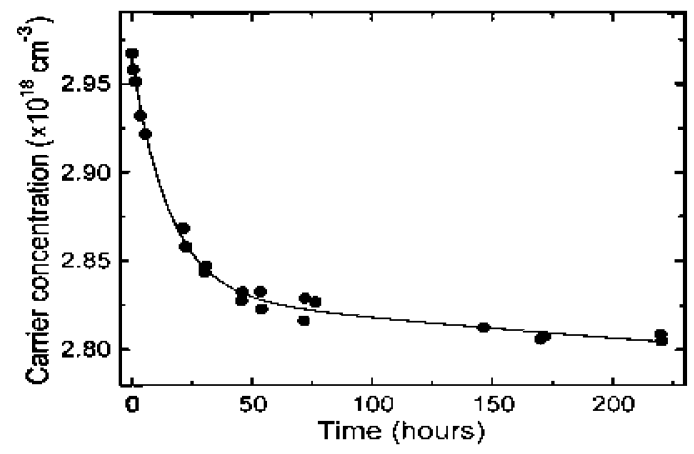

Figure 4 Electron concentration of $\mathrm{NC}$ a typical sample versus time after 1 minute immersion in $\mathrm{HCl}$.
4 Conclusion In summary, the Raman spectra of high quality InN nanocolumnar samples indicate that electron accumulation occurs at the non-polar lateral surfaces of the nanocolumns. This electron layer couples to the $\mathrm{E}_{1}(\mathrm{LO})$ phonon, giving rise to the observed mixed L. mode. The electron density depends on the growth temperature and is slightly sensitive to immersion in $\mathrm{HCl}$. The electron accumulation at the NC sidewalls is probably due to the formation of an In-rich surface layer during growth, as suggested by recent theoretical work.

Acknowledgements This work has been supported by research contracts of the Spanish Ministry of Education (MEC MAT2005-01388, NAN2004-09109-C04,TEC2004-05260-C0202, Consolider-CSD 2006-19) and the Community of Madrid (CAM S-0505-ESP-0200).

\section{References}

J. Grandal and M.A. Sánchez-García, J. Cryst. Growth 278, 373 (2005)

G. Abstreiter, M. Cardona, and A. Pinczuk, Light Scattering in Solids IV, edited by M. Cardona and G. Güntherodt (Springer-Verlag, Berlin, 1984), p. 5.

V. Yu. Davydov, V.V. Emtsev, I.N. Goncharuk, A.N. Smirnov, V.D. Petrikov, V.V. Mamutin, V.A. Vekshin, and S.N. Ivanov, Appl. Phys. Lett. 75, 3297 (1999); V. Yu. Davydov and A.A. Klochikhin, Semiconductors 38, 861 (2004).

T. Inushima, T. Shiraishia, and V.Yu. Davydov, Solid State Commun. 110, 491 (1999); T. Inushima, M. Higashiwaki, and T. Matsui, Phys. Rev. B 68, 235204 (2003).

A. Kasic, M. Schubert, Y. Saito, Y. Nanishi, and G. Wagner, Phys. Rev. B 65, 115206 (2002).

F. Demangeot, C. Pinquier, J. Frandon, M. Gaio, O. Briot, B. Maleyre, S. Ruffenach, and B. Gil, Phys. Rev. B 71, 104305 (2005)

J.S. Thakur, D. Haddad, V.M. Naik, R. Naik, G.W. Auner, H. Lu, and W.J. Schaff, Phys. Rev. B 71, 115203 (2005).

J.W. Pomeroy, M. Kuball, C.H. Swartz, T.H. Myers, H. Lu, and W.J. Schaff, Phys. Rev. B 75, 035205 (2007).

H.Y. Chen, C.H. Shen, H.W. Lin, C.H. Chen, C.Y. Wu, S. Gwo, V. Yu. Davydov, and A.A. Klochikhin, Thin Solid Films 515, 961 (2006).

I. Mahboob, T.D. Veal, C.F. McConville, H. Lu, and W.J. Schaff, Phys. Rev. Lett. 92, 36804-1 (2004).

H. Lu, W.J. Schaff, L.F. Eastman, and C.E. Stutz, Appl. Phys. Lett. 82, 1736 (2003).

L. Colakerol, T.D. Veal, H.K. Jeong, L. Plucinski, A. DeMasi, T. Learmonth, P.A. Glans, S. Wang, Y. Zhang, L.F.J. Piper, P.H. Jefferson, A. Fedorov, T.C. Chen, T.D. Moustakas, C.F. McConville, and K.E. Smith, Phys. Rev. Lett. 97, 237601 (2006)

D. Segev and C.G. Van de Walle, Europhys. Lett. 76(2), 305 (2006); Surf. Sci. 601, L-15 (2007); J. Cryst. Growth 300, 199 (2007).

J. Grandal, M.A.Sánchez-García, E. Calleja, E. Luna, and A. Trampert, Appl. Phys. Lett. 91, 021902 (2007).

Hai Lu, William J. Schaff, and Lester F. Eastman, J. Appl. Phys. 96, 3577 (2004). 